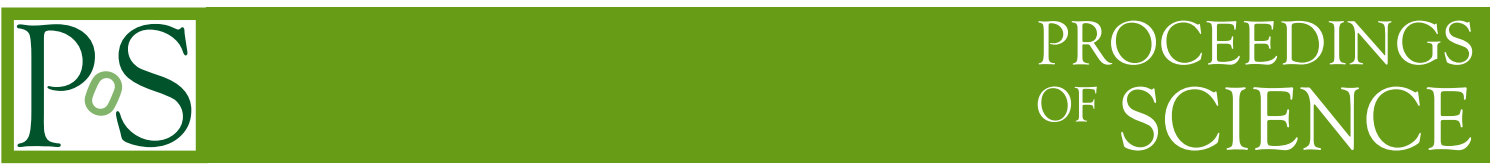

\title{
Correlations in small systems with ALICE
}

\section{Igor Lakomov (for the ALICE Collaboration)}

European Organization for Nuclear Research (CERN), CH-1211, Geneva 23, Switzerland

E-mail: igor.lakomov@cern.ch

\begin{abstract}
ALICE is dedicated to the study of the strongly interacting matter, the so-called Quark-Gluon Plasma (QGP), formed in heavy-ion collisions at the LHC. In addition, ALICE also actively participated in the $\mathrm{pp}$ and $\mathrm{p}-\mathrm{Pb}$ collision programs. In particular, the measurements of the twoparticle azimuthal correlations in pp collisions at $\sqrt{s}=7 \mathrm{TeV}$ and in $\mathrm{p}-\mathrm{Pb}$ collisions at $\sqrt{s_{\mathrm{NN}}}=$ $5.02 \mathrm{TeV}$ have been performed by the ALICE Collaboration during Run I of the LHC. Similar long-range correlations in $\mathrm{p}-\mathrm{Pb}$ and $\mathrm{Pb}-\mathrm{Pb}$ collisions have been observed on the near and away side - also known as the double ridge. Further investigations showed the importance of the Multi-Parton Interactions (MPI) in high-multiplicity collisions in small systems. In this work the ALICE results on the correlations in small systems are presented including MPI measurements in pp collisions.
\end{abstract}

38th International Conference on High Energy Physics

3-10 August 2016

Chicago, USA 


\section{Introduction}

In last few years all the four main LHC experiments performed many studies of azimuthal two-particle correlations in different collisions systems, from $\mathrm{pp}$ to $\mathrm{Pb}-\mathrm{Pb}$. It was seen that $\mathrm{a}$ near-side (around $\Delta \phi=0$ ) ridge is present in the data not only in $\mathrm{Pb}-\mathrm{Pb}$ collisions but also at high multiplicity in small systems. While in $\mathrm{Pb}-\mathrm{Pb}$ collisions it is a well-known feature which is usually explained by the presence of the anisotropic flow, appearance of this ridge in small systems raises a question about its origin: is it a result of initial or final state effects? While studying the multiplicity dependence of two-particle correlations in $\mathrm{p}-\mathrm{Pb}$ collisions, ALICE showed that peripheral $\mathrm{p}-\mathrm{Pb}$ collisions are similar to minimum bias pp collisions, where no near-side ridge is seen [1]. The associated-particle yield in peripheral $\mathrm{p}-\mathrm{Pb}$ collisions is then subtracted from that in central $\mathrm{p}-\mathrm{Pb}$ collisions to isolate the ridge from jet. After this procedure a "double-ridge" structure was observed which is shown in the right panel of the Fig. 1. This ridge was quantified in terms of $v_{\mathrm{n}}$ coefficients of the Fourier decomposition, and a clear mass ordering was found for $v_{2}$ which resembled $\mathrm{Pb}-\mathrm{Pb}$ case [2]. Such similar behaviour of central $\mathrm{p}-\mathrm{Pb}$ and $\mathrm{Pb}-\mathrm{Pb}$ collisions yields to further discussion on the possible collective effects in $\mathrm{p}-\mathrm{Pb}$ collisions. In this work we show a selection of the ALICE measurements on the correlations in small systems.
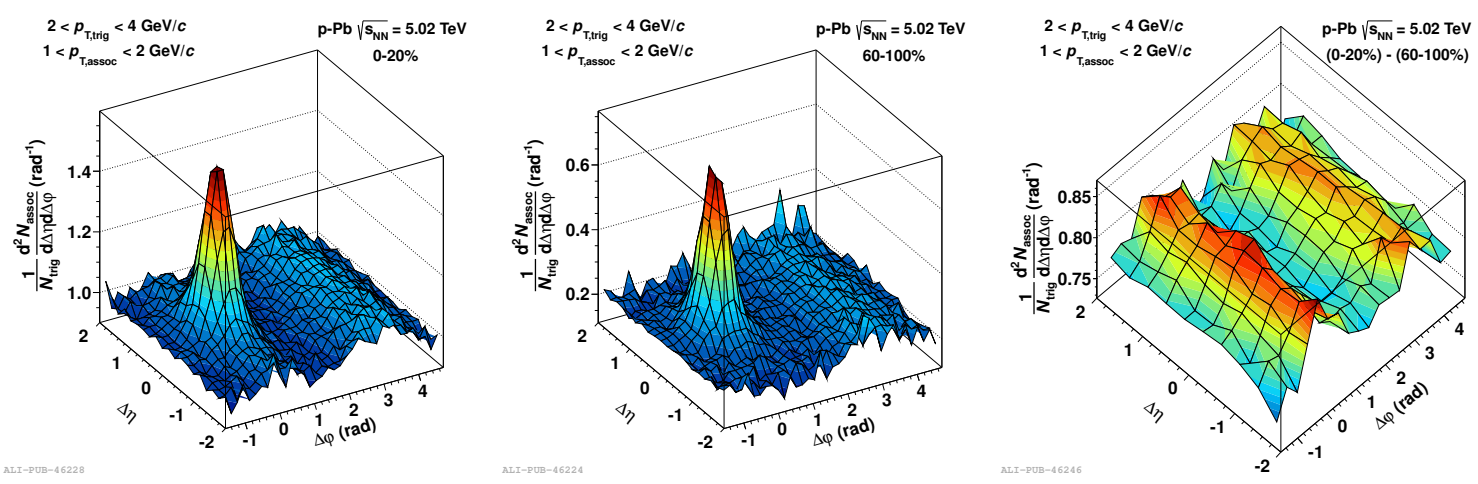

Figure 1: Double-ridge in $\mathrm{p}-\mathrm{Pb}$ collisions. The right panel with the double-ridge structure is the result of the subtraction of the scaled associated-particle yield for peripheral $\mathrm{p}-\mathrm{Pb}$ collisions (middle) from that for central p-Pb collisions (left).

\section{Long-range correlations in $\mathbf{p}-\mathrm{Pb}$ collisions}

In order to understand the origin of the observed double-ridge in $\mathrm{p}-\mathrm{Pb}$ collisions ALICE performed the study of the correlations between particles with a large pseudorapidity gap in $\mathrm{p}-\mathrm{Pb}$ collisions at $\sqrt{s_{\mathrm{NN}}}=5.02 \mathrm{TeV}$ [3]. For this study the muons detected by the ALICE forward muon spectrometer in pseudorapidity range $2.5<|\eta|<4$ were used as trigger particles, and associated particles were chosen from central barrel in pseudorapidity range $|\eta|<1.0$. The measurements have been performed in two configurations ( $\mathrm{p}-\mathrm{Pb}$, or $\mathrm{p}$-going direction, and $\mathrm{Pb}-\mathrm{p}$, or $\mathrm{Pb}$-going direction) which differ by the direction of the proton and lead beams with respect to the muon spectrometer. A double-ridge structure was observed in both configurations and it thus extends to large pseudorapidity, with $\eta$ reaching \pm 4 . The left and right panels of Fig. 2 show the Fourier decomposition of the observed double-ridge for $\mathrm{p}-\mathrm{Pb}$ and $\mathrm{Pb}-\mathrm{p}$ configurations, respectively. Second-order 
Fourier coefficient dominates in both cases. ALICE also measured $v_{2}$ of muons which is presented in Fig. 3. A sizeable muon $v_{2}$ is found for both $\mathrm{p}-\mathrm{Pb}$ and $\mathrm{Pb}-\mathrm{p}$ beam configurations which is larger in the latter case. The AMPT model shows a fair agreement with low- $p_{\mathrm{T}}$ measurements for $\mathrm{p}-\mathrm{Pb}$, while at higher $p_{\mathrm{T}}$ it fails to describe the data. The ratio of these two results is consistent with no $p_{\mathrm{T}}$-dependence. A possible mismatch of the AMPT compared to the data might be partly explained by the fact that the measurements are sensitive to the parent particle $v_{2}$ and composition of reconstructed muon tracks, where the contribution from heavy flavour decays are expected to dominate at $p_{\mathrm{T}}>2 \mathrm{GeV} / c$.
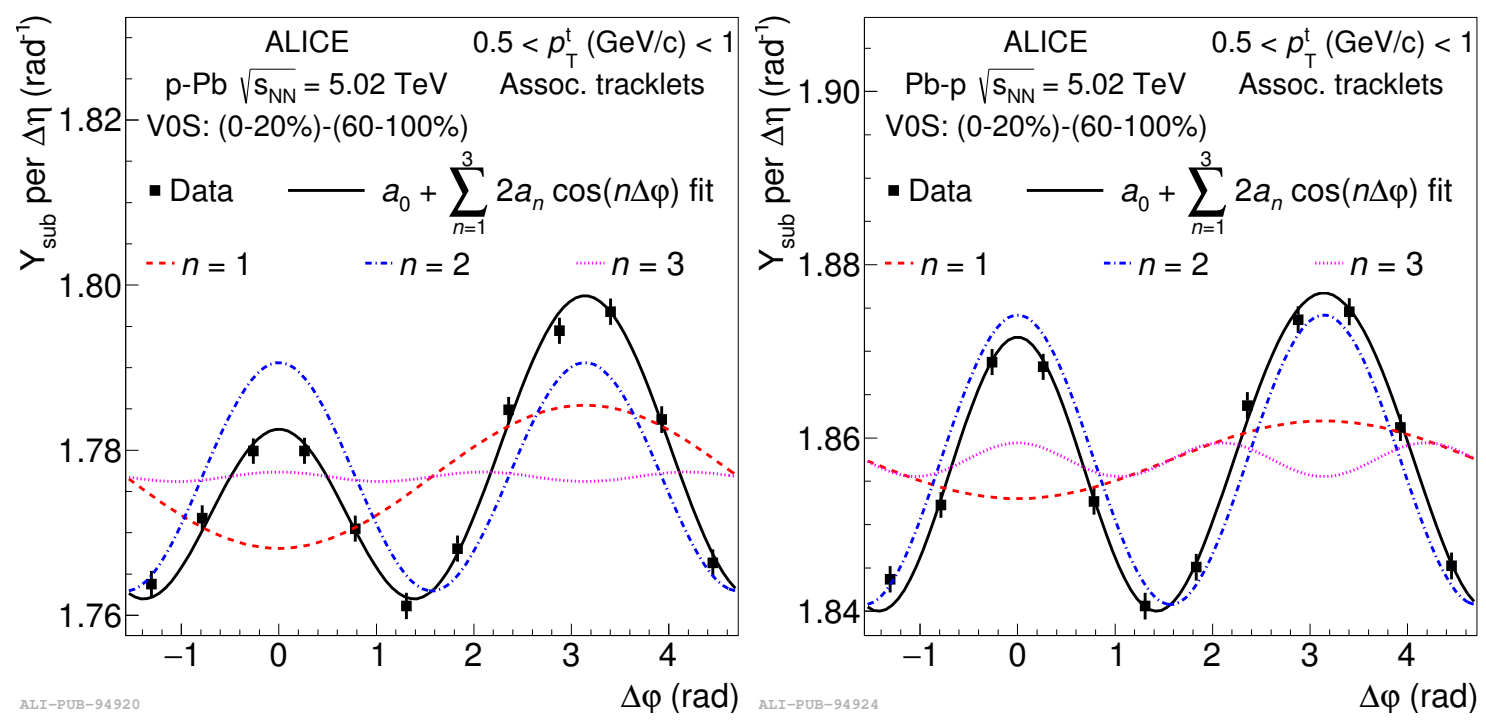

Figure 2: Fourier decomposition of the double-ridge in $\mathrm{p}-\mathrm{Pb}$ collisions for two configurations of the beams: $\mathrm{p}-\mathrm{Pb}$ (left) and $\mathrm{Pb}-\mathrm{p}$ (right).
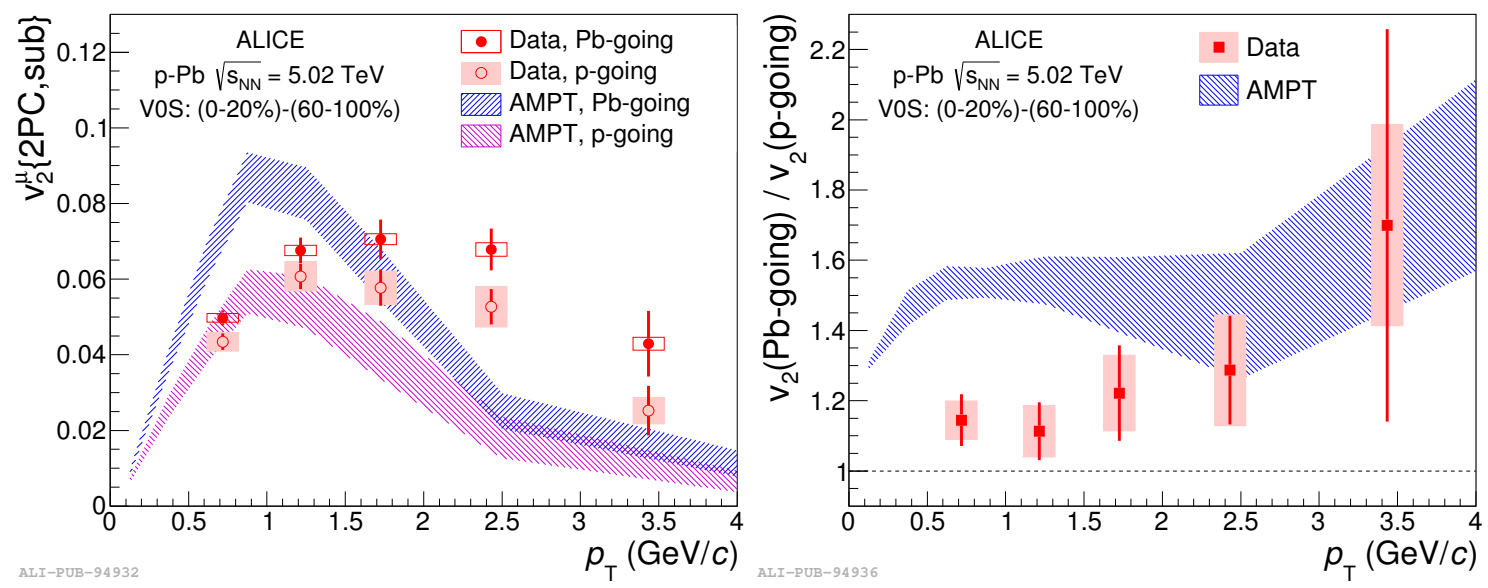

Figure 3: Muon $v_{2}$ in $\mathrm{p}-\mathrm{Pb}$ collisions as a function of $p_{\mathrm{T}}$ for 2 beam configurations (left) and their ratio (right). Open points in left panel represent the p-going configuration, while the filled points are for the $\mathrm{Pb}$-going configuration. 


\section{Multi-parton interactions in $\mathbf{p p}$ and $\mathbf{p}-\mathbf{P b}$ collisions}

Two-particle azimuthal correlations are also used to study multi-parton interactions (MPI). In PYTHIA generator $[4,5]$ the number of MPI is linearly proportional to the number of independent particle sources (number of uncorrelated seeds [6]), which can be measured in experiment: $\left\langle N_{\text {uncorr. seeds }}\right\rangle=\frac{\left\langle N_{\text {trigger }}\right\rangle}{1+\left\langle N_{\text {assoc, near+away }}\right\rangle}$, where $\left\langle N_{\text {trigger }}\right\rangle$ is the average number of trigger particles per event, $\left\langle N_{\text {assoc.,near+away }}\right\rangle$ is combined associated-particle yield in near (around $\Delta \phi=0$ ) and away (around $\Delta \phi=\pi$ ) sides. This quantity was measured by ALICE in pp at $\sqrt{s}=0.9,2.76$ and $7 \mathrm{TeV}[6]$ and in $\mathrm{p}-\mathrm{Pb}$ at $\sqrt{s_{\mathrm{NN}}}=5.02 \mathrm{TeV}$ [7]. The results are presented in Fig. 4 for pp (left panel) and $\mathrm{p}-\mathrm{Pb}$ (right panel) collisions. In all collisions systems and for different energies the number of uncorrelated seeds seems to grow linearly with the multiplicity with a hint of slight saturation at high multiplicity in pp collisions. This confirms the importance of the MPI in small systems. In right panel results are presented for two different $p_{\mathrm{T}}$ cuts. The number of uncorrelated seeds is higher for the wider $p_{\mathrm{T}}$ cut (going to lower $p_{\mathrm{T}}$ values). Higher reach in multiplicity at higher collision energy from Run II of the LHC data-taking period should provide a better understanding of the MPI at large energy densities.
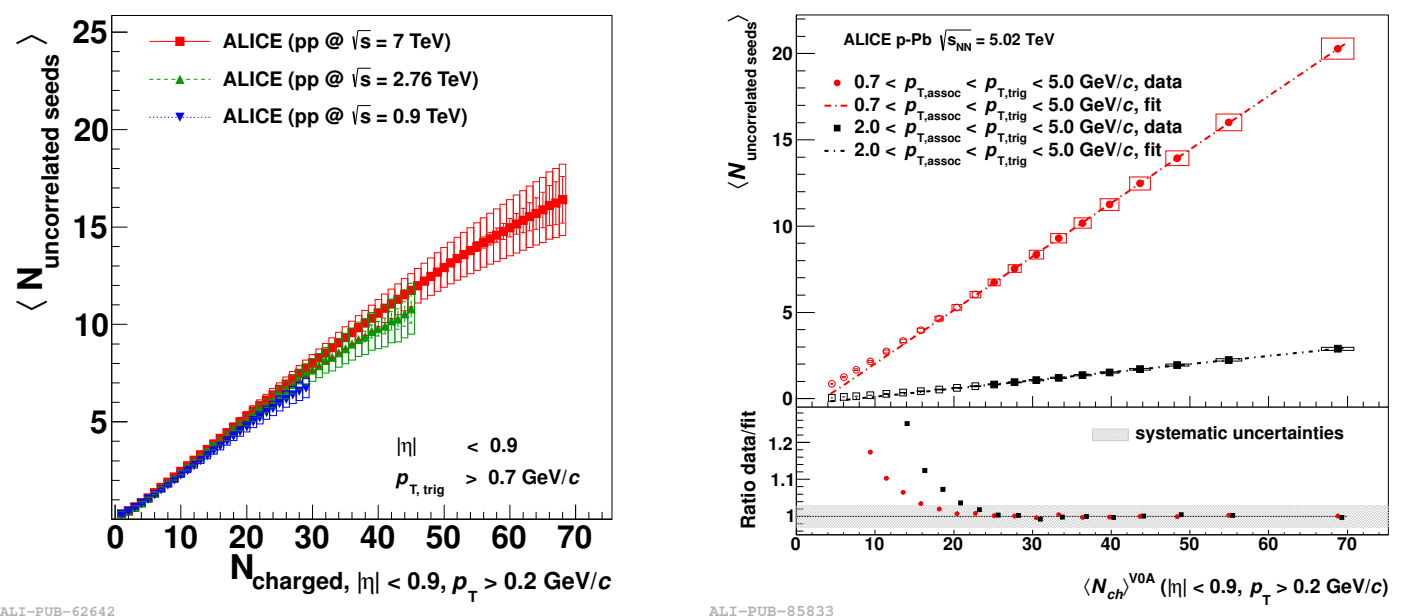

Figure 4: Uncorellated seeds as a function of multiplicity in $\mathrm{pp}$ (left) and in $\mathrm{p}-\mathrm{Pb}$ (right) collisions. In left panel results are presented for three energies with the same $p_{\mathrm{T}}$ cut for both trigger and associated particles $\left(p_{\mathrm{T}}>0.7 \mathrm{GeV} / c\right)$. In the right panel results for $\mathrm{p}-\mathrm{Pb}$ are given for two different $p_{\mathrm{T}}$ cuts $\left(0.7<p_{\mathrm{T}}<\right.$ $5.0 \mathrm{GeV} / c$ and $2.0<p_{\mathrm{T}}<5.0 \mathrm{GeV} / c$ for both trigger and associated particles).

\section{Summary}

ALICE has observed in $\mathrm{p}-\mathrm{Pb}$ collisions a double-ridge structure and a mass ordering in $v_{2}$ measurements. This might indicate some collective effects in $\mathrm{p}-\mathrm{Pb}$ collisions. Further studies of the muon-hadron correlations in $\mathrm{p}-\mathrm{Pb}$ showed that the double-ridge structure extends over 10 units of pseudorapidity. Inclusive muon $v_{2}$ has been measured to be larger on $\mathrm{Pb}$-going side than on $\mathrm{p}$ going side. Comparison of these measurements with the AMPT calculations suggests a non-zero $v_{2}$ 
of muon from heavy flavour decays. Finally, the number of correlated seeds (linearly proportional to the number of MPI) was found to scale linearly with multiplicity both in $\mathrm{pp}$ and $\mathrm{p}-\mathrm{Pb}$ collisions at different energies. Further measurement at higher energies and with higher multiplicity reach will provide better understanding of MPI in small systems.

\section{References}

[1] ALICE collaboration, B. Abelev et al., Long-range angular correlations on the near and away side in $p-P b$ collisions at $\sqrt{s_{N N}}=5.02$ TeV, Phys. Lett. B719 (2013) 29-41, [1212.2001].

[2] ALICE collaboration, E. Abbas et al., Centrality dependence of the pseudorapidity density distribution for charged particles in Pb-Pb collisions at $\sqrt{s_{\mathrm{NN}}}=2.76$ TeV, Phys. Lett. B726 (2013) 610-622, [1304.0347].

[3] ALICE collaboration, J. Adam et al., Forward-central two-particle correlations in $p$-Pb collisions at $\sqrt{s_{\mathrm{NN}}}=5.02 \mathrm{TeV}$, Phys. Lett. B753 (2016) 126-139, [1506.08032].

[4] T. Sjostrand, S. Mrenna and P. Z. Skands, PYTHIA 6.4 Physics and Manual, JHEP 05 (2006) 026, [hep-ph/0603175].

[5] T. Sjostrand, S. Mrenna and P. Z. Skands, A Brief Introduction to PYTHIA 8.1, Comput. Phys. Commun. 178 (2008) 852-867, [0710.3820].

[6] ALICE collaboration, B. Abelev et al., Multiplicity dependence of two-particle azimuthal correlations in pp collisions at the LHC, JHEP 09 (2013) 049, [1307 . 1249].

[7] ALICE collaboration, B. B. Abelev et al., Multiplicity dependence of jet-like two-particle correlation structures in p-Pb collisions at $\sqrt{s_{N N}}=5.02 \mathrm{TeV}$, Phys. Lett. B741 (2015) 38-50, [1406.5463]. 